Pacific Journal of Mathematics

SOME RING EXTENSIONS WITH MATRIX

CARL W. KOHLS AND LAWRENCE JAMES LARD 


\title{
SOME RING EXTENSIONS WITH MATRIX REPRESENTATIONS
}

\author{
C. W. KoHLS AND L. J. LARDY
}

\begin{abstract}
A new type of ring extension introduced by $J$. Szendrei, which may be viewed as a generalization of the splitting Everett extension, will be discussed. The examples of this type of extension given by Szendrei are generalizations of the complex field extension of the real field, and of the quaternion extension of the complex field. Our investigation produces a larger class of examples.
\end{abstract}

In [4], J. Szendrei considered a very general type of ring extension. One of the four special cases that he lists is the type that will be studied further in this paper. We shall formulate the definition of the extension in terms of bimultiplications (cf. [2] or [3]). A bimultiplication $\sigma$ of a ring $A$ is a pair of mappings $a \rightarrow \sigma a$, $a \rightarrow a \sigma$ of $A$ into itself satisfying the rules

$$
\sigma(a+b)=\sigma a+\sigma b,(a+b) \sigma=a \sigma+b \sigma, \sigma(a b)=(\sigma a) b,(a b) \sigma=a(b \sigma),
$$

and $a(\sigma b)=(a \sigma) b$, for all $a, b \in A$. A pair of bimultiplications $\sigma$ and $\tau$ is said to be permutable if $\sigma(a \tau)=(\sigma a) \tau$ and $\tau(a \sigma)=(\tau a) \sigma$ for all $a \in A$, and a set of bimultiplications is permutable if every pair in the set is permutable. The sum $\sigma+\tau$ and the product $\sigma \tau$ of two bimultiplications are defined by the equations $(\sigma+\tau) a=\sigma a+\tau a$, $a(\sigma+\tau)=a \sigma+a \tau,(\sigma \tau) a=\sigma(\tau a)$, and $a(\sigma \tau)=(a \sigma) \tau$ for all $a \in A$. Under these operations the set of all bimultiplications of $A$ is a ring, denoted by $M_{A}$. For each element $c$ of $A$, a bimultiplication $\nu_{c}$ is obtained by setting $\nu_{c} a=c a$ and $a \nu_{c}=a c$ for all $a \in A$. Clearly the mapping $\nu: A \rightarrow M_{A}$, defined by $c \rightarrow \nu_{c}$, is a ring homomorphism. Bimultiplications in the range of $\nu$ are called inner bimultiplications.

Let $A$ and $B$ be rings. We define the ring $A * B$ to be the direct sum of $A$ and $B$ as additive groups, with multiplication given by

$$
(a, b)(c, d)=\left(a c+\{b, d\}, \sigma_{a} d+b \sigma_{c}+b d\right),
$$

where $\sigma$ is a homomorphism from $A$ onto a ring of permutable bimultiplications of $B$, and $\{\cdot, \cdot\}$ is a bilinear function from $B \times B$ into $A$ satisfying the equations

$$
\begin{aligned}
b \sigma_{\{c, d\}} & =\sigma_{\{b, c\}} d, \\
\{b, c d\} & =\{b c, d\}, \\
\left\{b, \sigma_{a} c\right\} & =\left\{b \sigma_{a}, c\right\},
\end{aligned}
$$




$$
\left\{\sigma_{a} b, c\right\}=a\{b, c\}, \quad \text { and } \quad\left\{b, c \sigma_{a}\right\}=\left\{b, c^{\prime} a,\right.
$$

for all $a \in A$ and all $b, c, d \in B$. The mapping $a \rightarrow(a, 0)$ is a monomorphism of $A$ into the ring $A * B$. In case the bilinear function $\{\cdot, \cdot\}$ is identically zero, the extension reduces to a splitting Everett extension [2]. However, in contrast to the Everett extension, the ring $A * B$ need not contain an ideal isomorphic with $B$.

Note that if $B$ has an identity, then (1.4) is redundant: By (1.3), $\{c, d\}=\{1, c d\}$, so $\left\{b, \sigma_{a} c\right\}=\left\{1, b\left(\sigma_{a} c\right)\right\}$ and $\left\{b \sigma_{a}, c\right\}=\left\{1,\left(b \sigma_{a}\right) c\right\}$. But $b\left(\sigma_{a} c\right)=\left(b \sigma_{a}\right) c$, since $\sigma_{a}$ is a bimultiplication, so (1.4) is satisfied.

Let $p$ be a fixed element in the center of $A$. The ring whose additive group coincides with that of $A$ and whose multiplication is defined by the mapping $(a, b) \rightarrow p a b$ will be denoted by $A_{p}$. We shall not introduce a new symbol for the product in $A_{p}$; products can be written out in terms of the given multiplication in $A$. Our discussion is concerned with extensions of the form $A * A_{p}$.

We denote the inverse of an automorphism $\phi$ by $\dot{\phi}^{\prime}$, and the identity automorphism by $I$.

2. The main results. We begin this section with some elementary results concerning the particular types of functions $\sigma$ and $\{\cdot, \cdot\}$ that will be used later.

Proposition 1. Let $A$ be a ring and let $\phi$ and $\psi$ be any maps from $A$ to $A$ such that $\phi(a)-\psi(a)$ is in the annihilator of $A_{p}$ for all $a \in A$. Define $\sigma$ on $A$ by

$$
\sigma_{a} b=\phi(a) b, b \sigma_{a}=b \psi(a)
$$

for all $a, b \in A$.

Then $\sigma$ maps onto a set of permutable bimultiplications of $A_{p}$, and is a homomorphism if both $\phi$ and $\psi$ are homomorphisms.

Proof. Since $p$ lies in the center of $A$, the fact that $\sigma$ maps onto a set of permutable bimultiplications of $A_{p}$ follows directly from the assumption that $\phi(a)-\psi(a)$ is in the annihilator of $A_{p}$ together with the associative and distributive laws for $A$. These same laws ensure that $\sigma$ is a homomorphism if both $\phi$ and $\psi$ are homomorphisms.

Note that, in general, the bimultiplications of $A_{p}$ defined by (2.1) are not inner bimultiplication. For example, if $p=0$ then there is only one inner bimultiplications. Indeed, when $\phi$ and $\psi$ are homomorphisms, we in effect make $A_{p}$ into an $A$-bimodule and let the actions of $A$ on $A_{p}$ determine the range of $\sigma$.

Proposition 2. Let $A$ be a ring with identity and let $\phi$ and $\psi$ 
be automorphisms of $A$. Suppose that for a given extension $A * A_{p}$, $\sigma$ is defined by (2.1). Then, for some $q \in A$,

$$
\{a, b\}=\phi^{\prime}(a) q \psi^{\prime}(b)
$$

for all $a, b \in A$.

Conversely, suppose that for a given extension $A * A_{p},\{\cdot, \cdot\}$ is defined by (2.2), where $q$ is not a zero-divisor. Then $\sigma$ is given by (2.1).

Proof. By (1.5), $\phi^{\prime}(a)\{1,1\} \psi^{\prime}(b)=\left\{\sigma_{\phi^{\prime}(a)} 1,1 \sigma_{\psi^{\prime}(b)}\right\}=\{a 1,1 b\}=\{a, b\}$. The result follows with $q=\{1,1\}$.

For the converse, using (1.5) again,

$$
\phi^{\prime}\left(\sigma_{a} b\right) q \psi^{\prime}(1)=\left\{\sigma_{a} b, 1\right\}=a\{b, 1\}=a \phi^{\prime}(b) q \psi^{\prime}(1) .
$$

Thus, since $q$ is not a zero-divisor, $\phi^{\prime}\left(\sigma_{a} b\right)=a \phi^{\prime}(b)$ and $\sigma_{a} b=\phi(a) b$. The proof of the other equation is similar.

For the remaining discussion we assume that $q$ is in the center of $A$. We now give conditions relating $\phi, \psi, p$, and $q$ such that equations (2.1) and (2.2) determine an extension $A * A_{p}$. Under these conditions, we then represent the extension as a subring of the ring of $2 \times 2$ matrices over $A$.

THEOREM. Let $\phi$ and $\psi$ be automorphisms of a ring $A$, and let $q$ be a fixed element in the center of $A$. Define $\sigma$ and $\{\cdot, \cdot\}$ by (2.1) and (2.2) respectively. Assume that

(2.3) $\dot{\phi}(a)-\psi(a)$ is in the annihilator of $A_{p}$ for all $a \in A$,

(2.4) $\phi^{\prime}=\psi^{\prime}$ on $\{p a: a \in A\}$,

(2.5) $\phi(q)=\psi(q)$,

and

(2.6) $\phi^{\prime} \psi=\psi^{\prime} \phi$.

Then with multiplication defined by (1.1), one obtains a ring extension $A * A_{p}$, and it is isomorphic with a subring of the ring of $2 \times 2$ matrices over $A$. The isomorphism is given by

$$
(a, b) \rightarrow\left[\begin{array}{cc}
a & \phi^{\prime}(b) \\
q \psi^{\prime}(b) & \phi^{\prime}(\psi(a)+p b)
\end{array}\right], \quad \quad a, b \in A .
$$

Conversely, if $A * A_{p}$ is a ring extension and $A$ has an element that is not a zero-divisor, then (2.3) holds. If $q$ is not a zero-divisor, then (2.4), (2.5), and (2.6) also hold.

Proof. Since $\phi$ and $\psi$ are automorphisms and (2.3) holds, it 
follows from Proposition 1 that $\sigma$ is a homomorphism from $A$ onto a ring of permutable bimultiplications of $A_{p}$. Clearly $\{\cdot, \cdot\}$ is bilinear; so a ring extension $A * A_{p}$ will be obtained if equations (1.2)-(1.5) are satisfied. Equation (1.2) can be verified using (2.5) and (2.6). Equation (1.3) follows from (2.4) and the definition of multiplication in $A_{p}$. Equation (1.4) follows immediately from (2.6). Finally, (1.5) is a direct consequence of (2.1) and (2.2).

The isomorphism follows from a straightforward albeit cumbersome calculation using (2.3)-(2.6) and the definition of multiplication in $A_{p}$.

For the necessity, condition (2.3) is a consequence, under the stated hypothesis, of the fact that $\left(a \sigma_{b}\right) c=a\left(\sigma_{b} c\right)$ for all $a, b, c \in A$. If $q$ is not a zero-divisor, then, since $q$ is in the center of $A,(2.4)$ follows from (1.3), (2.6) follows from (1.4), and (2.5) follows from (1.2) and (2.6) combined.

CoROllaRy 1. If $q$ is not a zero-divisor, then (2.1) and (2.2) determine an extension $A * A_{p}$ if and only if (2.3)-(2.6) hold.

Corollary 2. If $p=0$ and (2.5)-(2.6) hold, then one obtains a ring extension $A * A_{0}$, with matrix representation

$$
(a, b) \rightarrow\left[\begin{array}{cc}
a & \phi^{\prime}(b) \\
q \psi^{\prime}(b) & \phi^{\prime} \psi(a)
\end{array}\right]
$$

CoRollary 3. If $\phi=\psi$, then one obtains a ring extension $A * A_{p}$, with matrix representation

$$
(a, b) \rightarrow\left[\begin{array}{cc}
a & \phi^{\prime}(b) \\
q \phi^{\prime}(b) & a+\phi^{\prime}(p b)
\end{array}\right] .
$$

Moreover, $A * A_{p}$ is isomorphic with the extension $A * A_{\phi^{\prime}(p)}$, where (2.1) and (2.2) are defined with the identity automorphism and the same $q$ as in the extension $A * A_{p}$.

Proof. The first statement follows immediately from the theorem. To establish the isomorphism, observe that the product in $A * A_{\phi^{\prime}(p)}$ is given by

$$
(a, b)(c, d)=\left(a c+q b d, a d+b c+\phi^{\prime}(p) b d\right),
$$

while the product in $A * A_{p}$ is given by

$$
(a, b)(c, d)=\left(a c+q \phi^{\prime}(b d), \phi(a) d+b \phi(c)+p b d\right) .
$$

The mapping $A * A_{\phi^{\prime}}(p) \rightarrow A * A_{p}$ defined by $(a, b) \rightarrow(a, \phi(b))$ is then 
easily seen to be the desired isomorphism.

REMARK 1. In connection with Corollary 3 we note that if $\dot{\phi}=\psi$ and $A$ is commutative, then $A * A_{p}$ is commutative. Also, if the extension $A * A_{p}$ is commutative, then of course $A$ must be commutative; if in addition, $A$ contains an element that is not a zero-divisor, then we can deduce that $\phi=\psi$ as well.

Remark 2. Let $A$ be a field, and $\alpha \in A * A_{p}$. We may view $\alpha$ as the $2 \times 2$ matrix associated with it by the theorem. Now if $\operatorname{det} \alpha \neq 0$, then of course $\alpha$ is a unit in the ring of $2 \times 2$ matrices over $A$; a routine verification shows that $\alpha$ is actually a unit in $A * A_{p}$.

ExAmple 1. Let $S$ be a square in the plane, let $A$ be the ring of all continuous real-valued functions on $S$, and let $\phi$ and $\psi$ be the automorphisms induced by the reflections of $S$ in its diagonals. Then, since the reflections commute and have period two, $\dot{\phi}^{2}=\psi^{2}=I$ and $\phi \psi=\psi \phi$, so (2.6) is satisfied. If $q=1$ and $p=0$, then by Corollary $2, A * A_{0}$ is an extension. Let $a \in A$ be a nonzero function such that $\phi(a)=-a$ and $\psi(a)=a$. Then $(a, a)^{2}=(0,0)$ in $A * A_{0}$. Thus the extension is not a ring of functions with pointwise operations.

Example 2. Let $A$ be the direct sum of two copies of the complex field $C, p=(0,1), q=(0,0), \phi(a, b)=(\bar{a}, b)$, and $\psi=I$. Then (2.3)-(2.6) are satisfied, but $p \neq 0$ and $\phi \neq \psi$. This extension is isomorphic to a direct sum of two extensions of $C$ by itself. In the first extension we have conjugation for one automorphism and $I$ for the other, with $p=q=0$; in the second extension we have $I$ for both automorphisms, with $p=1$ and $q=0$.

EXAMPLE 3. If $\phi=I, \psi^{2}=I, p=0$, and $\psi(q)=q$, then conditions (2.5)-(2.6) are satisfied, and $A * A_{0}$ is a "quaternion" extension isomorphic with the ring of matrices of the form

$$
\left[\begin{array}{cc}
a & b \\
q \psi(b) & \psi(a)
\end{array}\right]
$$

Szendrei's "quaternion" extensions are all of this form. However, the extension of the four element field $\{0,1, \theta, 1+\theta\}$ obtained with $\psi(\theta)=1+\theta$ and $q=1$, is not a Szendrei "quaternion" extension.

As a special case, let $A$ be the field obtained from a totally ordered field $F$ by adjoining the square root of a negative element $f \in F$, define $\psi$ by $\psi \mid F=I, \psi(\sqrt{f})=-\sqrt{f}$, and let $q=-1$. Then $a \psi(a)>0$ for all nonzero $a$ in $A$, and one can show immediately, 
using Remark 2, that the extension is a division ring. Also, it is easy to see that if $a \psi(a)>0$ for all nonzero $a$ in $A$ and $\psi^{2}=I$, then $\psi$ must be as defined.

Example 4. If $\phi=\psi$ and $A$ has an identity, then the extension of Corollary 3 contains a root of the quadratic equation $x^{2}-\phi^{\prime}(p) x-q=0$, namely,

$$
\left[\begin{array}{cc}
0 & 1 \\
q & \phi^{\prime}(p)
\end{array}\right]
$$

When $\phi=\psi=I, p=0$, and $q=-1$, we recover Szendrei's "complex" extension.

3. Some special cases. This section contains a discussion of extensions of the form $A * A_{p}$ when $A$ is a field or an integral domain, with the additional assumption that $\phi=\psi$. Thus the representation is that given by Corollary 3. In view of Corollary 3, we can assume without loss of generality that $\phi=\psi=I$.

Proposition 3. Let $A$ be a field. If $x^{2}-p x-q$ is irreducible over $A$, then $A * A_{p}$ is a field. If $x^{2}-p x-q$ has a root in $A$, and $p^{2}+4 q=0$, then $A * A_{p}$ has a basis (as a 2-dimensional algebra over $A$ ) consisting of the identity and a nilpotent element of index two. If $x^{2}-p x-q$ has a root in $A$ and $p^{2}+4 q \neq 0$, then $A * A_{p}$ is isomorphic with $A \oplus A$.

Proof. The determinant of the element

$$
\left[\begin{array}{cc}
a & b \\
q b & a+p b
\end{array}\right]
$$

is $a^{2}+p a b-q b^{2}$. If $x^{2}-p x-q$ is irreducible and the characteristic of $A$ is not two, then $p^{2}+4 q$ is not a square and we can write

$$
a^{2}+p a b-q b^{2}=(a+p b / 2)^{2}-\left(p^{2}+4 q\right) b^{2} / 4 ;
$$

if the characteristic of $A$ is two, we note that $a^{2}+p a b-q b^{2}$ is $a^{2}$ when $b=0$ and $b^{2}\left[(a / b)^{2}-p(a / b)-q\right]$ when $b \neq 0$. In either case, it follows quickly that the determinant is zero if and only if both $a$ and $b$ are zero. Thus, by Remark 2 , we see that $A * A_{p}$ is a field.

Now assume that $x^{2}-p x-q$ has a root in $A$ and $p^{2}+4 q=0$. If the characteristic of $A$ is not two, then $\left[\begin{array}{cc}-p / 2 & 1 \\ q & p / 2\end{array}\right]$ is in $A * A_{p}$ and is nilpotent of index two. If the characteristic of $A$ is two, then $p=0$, so there exists $c \in A$ such that $c^{2}=q$. Then $\left[\begin{array}{ll}c & 1 \\ q & c\end{array}\right]$ is in 
$A * A_{p}$ and is nilpotent of index two. Clearly the identity and the indicated nilpotent elements are linearly independent, and thus form a basis for $A * A_{p}$.

Finally, assume that $x^{2}-p x-q$ has a root in $A$ and $p^{2}+4 q \neq 0$. If the characteristic of $A$ is not two, then $p^{2}+4 q$ is a square; let $d \in A$ satisfy $d^{2}\left(p^{2}+4 q\right)=1$. Then the element

$$
\alpha_{1}=\left[\begin{array}{cc}
(1-p d) / 2 & d \\
q d & (1+p d) / 2
\end{array}\right]
$$

is a nontrivial idempotent. If the characteristic of $A$ is two, let $a$ be a root of $x^{2}-p x-q$ in $A$, and set

$$
\alpha_{2}=\left[\begin{array}{cc}
p^{-1} a & p^{-1} \\
q p^{-1} & p^{-1} a+1
\end{array}\right] \text {. }
$$

Then $\alpha_{2}$ is a nontrivial idempotent. The isomorphism follows quickly since $\alpha_{i}$ and $1-\alpha_{i}$, for $i=1,2$, are orthogonal idempotents.

REMARK 3. If the characteristic of $A$ is not two, we can replace the hypotheses by: $p^{2}+4 q$ is not a square, $p^{2}+4 q=0$, and $p^{2}+4 q$ is a nonzero square, respectively.

REMARK 4. Of course, in general, the various field extensions, obtained in part one will not be isomorphic.

Remark 5. If $A$ is the complex field and $\phi=\psi$, then $A * A_{p}$ is not a field regardless of the choice of $p$ and $q$. However, it is possible to obtain the division ring of quaternions as an extension of the complex field with $\phi \neq \psi$, as indicated in Example 3.

REMark 6. Since an extension $A * A_{1}$, where $A$ is the complex field, can contain nontrivial nilpotent elements, it follows that semisimplicity is not, in general, inherited by the extension. We note that for commutative rings, semisimplicity is inherited by Everett extensions [1, Th. 4].

Proposition 4. Let $A$ be an integral domain. Then $A * A_{p}$ is an integral domain if and only if $x^{2}-p x-q$ is irreducible over the quotient field of $A$.

Proof. Assume that $x^{2}-p x-q$ is irreducible over the quotient field $F$ of $A$. Let $\alpha \in A * A_{p}, \alpha \neq 0$. View $\alpha$ as an element of $F * F_{p}$. Then, by Proposition 3 , $\operatorname{det} \alpha \neq 0$ and $\alpha^{-1}$ exists in $F * F_{p}$. Since $(\operatorname{det} \alpha) \alpha^{-1} \in A * A_{p}$, if $\alpha \beta=0$, then $(\operatorname{det} \alpha) \beta=0$. But $A$ is an integral 
domain, so $\beta=0$. Thus, $A * A_{p}$ is an integral domain.

If $x^{2}-p x-q$ is not irreducible over $F$, then by Proposition 3 , $F * F_{p}$ contains either a nontrivial idempotent or a nontrivial nilpotent element of index two. If $\beta$ is such an element in $F * F_{p}$, then for suitable nonzero $b \in A$ we have $b \beta \in A * A_{p}$. Thus, $b \beta$ is a zero-divisor in $A * A_{p}$.

\section{REFERENCES}

1. C. W. Kohls, Properties inherited by ring extensions. Michigan Math. J. 12 (1965), 399-404.

2. C. W. Kohls and L. J. Lardy, On extensions and bimultiplication algebras of algebras, (submitted for publication).

3. S. MacLane, Extensions and obstructions for rings, Illinois J. Math. 2 (1958), 316345.

4. J. Szendrei, Über eine allgemeine Ringkonstruktion durch schiefes Produkt, Acta Sci. Math. 19 (1958), 63-76.

Received October 3, 1966.

SYRACUSE UNIVERSITY 


\title{
PACIFIC JOURNAL OF MATHEMATICS
}

\author{
EDITORS
}

\author{
H. ROYDEN \\ Stanford University \\ Stanford, California
}

\author{
J. P. JANS \\ University of Washington \\ Seattle, Washington 98105
}

\section{J. DUGUNDJI}

Department of Mathematics University of Southern California Los Angeles, California 90007

\section{RICHARD ARENS}

University of California Los Angeles, California 90024

\section{ASSOCIATE EDITORS}

\author{
E. F. BECKENBACH
}

B. H. NEUMANN

F. WoLF

K. YosidA

\section{SUPPORTING INSTITUTIONS}

\author{
UNIVERSITY OF BRITISH COLUMBIA \\ CALIFORNIA INSTITUTE OF TECHNOLOGY \\ UNIVERSITY OF CALIFORNIA \\ MONTANA STATE UNIVERSITY \\ UNIVERSITY OF NEVADA \\ NEW MEXICO STATE UNIVERSITY \\ OREGON STATE UNIVERSITY \\ UNIVERSITY OF OREGON \\ OSAKA UNIVERSITY \\ UNIVERSITY OF SOUTHERN CALIFORNIA
}

\author{
STANFORD UNIVERSITY \\ UNIVERSITY OF TOKYO \\ UNIVERSITY OF UTAH \\ WASHINGTON STATE UNIVERSITY \\ UNIVERSITY OF WASHINGTON \\ AMERICAN MATHEMATICAL SOCIETY \\ CHEVRON RESEARCH CORPORATION \\ TRW SYSTEMS \\ NAVAL WEAPONS CENTER
}

Mathematical papers intended for publication in the Pacific Journal of Mathematics should be in typed form or offset-reproduced, double spaced with large margins. Underline Greek letters in red, German in green, and script in blue. The first paragraph or two must be capable of being used separately as a synopsis of the entire paper. It should not contain references to the bibliography. Manuscripts, in duplicate if possible, may be sent to any one of the four editors. All other communications to the editors should be addressed to the managing editor, Richard Arens, University of California, Los Angeles, California 90024.

Each author of each article receives 50 reprints free of charge; additional copies may be obtained at cost in multiples of 50 .

The Pacific Journal of Mathematics is published monthly. Effective with Volume 16 the price per volume (3 numbers) is $\$ 8.00$; single issues, $\$ 3.00$. Special price for current issues to individual faculty members of supporting institutions and to individual members of the American Mathematical Society: $\$ 4.00$ per volume; single issues $\$ 1.50$. Back numbers are available.

Subscriptions, orders for back numbers, and changes of address should be sent to Pacific Journal of Mathematics, 103 Highland Boulevard, Berkeley 8, California.

Printed at Kokusai Bunken Insatsusha (International Academic Printing Co., Ltd.), 7-17, Fujimi 2-chome, Chiyoda-ku, Tokyo, Japan.

PUBLISHED BY PACIFIC JOURNAL OF MATHEMATICS, A NON-PROFIT CORPORATION

The Supporting Institutions listed above contribute to the cost of publication of this Journal, but they are not owners of publishers and have no responsibility for its content or policies. 


\section{Pacific Journal of Mathematics}

Vol. 26, No. 2 December, 1968

Seymour Bachmuth and Horace Yomishi Mochizuki, Kostrikin's theorem on

Engel groups of prime power exponent ....................

Paul Richard Beesack and Krishna M. Das, Extensions of Opial's inequality ...................................... 215

John H. E. Cohn, Some quartic Diophantine equations .............. 233

H. P. Dikshit, Absolute $(C, 1) \cdot\left(N, p_{n}\right)$ summability of a Fourier series and its conjugate series ............................... 245

Raouf Doss, On measures with small transforms ................. 257

Charles L. Fefferman, $L_{p}$ spaces over finitely additive measures........ 265

Le Baron O. Ferguson, Uniform approximation by polynomials with integral coefficients. II...................................

Takashi Ito and Thomas I. Seidman, Bounded generators of linear

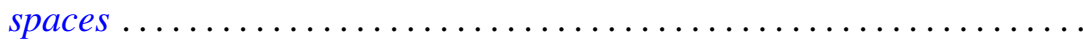

Masako Izumi and Shin-ichi Izumi, Nörlund summability of Fourier series ..........................................

Donald Gordon James, On Witt's theorem for unimodular quadratic

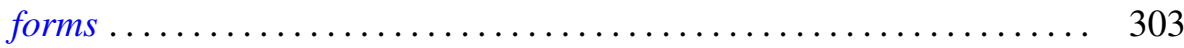

J. L. Kelley and Edwin Spanier, Euler characteristics .............. 317

Carl W. Kohls and Lawrence James Lardy, Some ring extensions with matrix

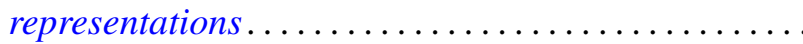

Ray Mines, III, A family of functors defined on generalized primary groups ....

Louise Arakelian Raphael, A characterization of integral operators on the space of Borel measurable functions bounded with respect to a weight function....

Charles Albert Ryavec, The addition of residue classes modulo $n .$.

H. M. (Hari Mohan) Srivastava, Fractional integration and inversion formulae associated with the generalized Whittaker transform ...

Edgar Lee Stout, The second Cousin problem with bounded data ...

Donald Curtis Taylor, A generalized Fatou theorem for Banach

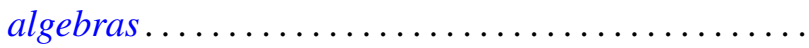

Bui An Ton, Boundary value problems for elliptic convolution equations of Wiener-Hopf type in a bounded region...

Philip C. Tonne, Bounded series and Hausdorff matrices for absolutely convergent sequences... 